\title{
Time Series Analysis of Grey Forecasting Based on Wavelet Transform and Its Prediction Applications
}

\author{
Haiyan Cen, Yidan Bao, Min Huang, and Yong He \\ College of Biosystems Engineering and Food Science, Zhejiang University, 310029, \\ Hangzhou, China \\ yhe@zju.edu.cn
}

\begin{abstract}
Grey forecasting based on GM $(1,1)$ has become an important methodology in time series analysis. But due to the limitation of predicting nonstationary time series, an improved grey forecasting GM $(1,1)$ model with wavelet transform was proposed. The time series data was first decomposed to different scales by wavelet transform with à trous algorithm previous of Mallat algorithm in the parallel movement of time series, and then the decomposed time series were forecasted by GM $(1,1)$ model to obtain forecasting results of the original time series. Time series prediction capability of GM $(1,1)$ combined with wavelet transform was compared with that of traditional GM $(1,1)$ model and autoregressive integrated moving average (ARIMA) model to energy source consumption and production forecasting in China. To effectiveness of these methods, eighteen years of time series records (1985 to 2002) for energy source consumption and production were used. The forecasting result from GM $(1,1)$ model with wavelet transform for the data from 2000 to 2002 presented highest precision of three models. It shows that the GM $(1,1)$ model with wavelet transform is more accurate and performs better than traditional GM $(1,1)$ and ARIMA model.
\end{abstract}

\section{Introduction}

Time series analysis was used to forecast the developing trend or changes in the future according to a data set arranged by time, and it has been applied widely in many different fields such as economics, sociology and science. Traditional statistical models including autoregressive (AR), moving average (MA), exponential smoothing, autoregressive moving average (ARMA) and autoregressive integrated moving average (ARIMA) [1] are the most popular time series methodologies, but their forecasting abilities are constrained by their assumption of a linear behavior and thus it is not very satisfactory. To improve forecasting non-linear time series events, alternative modeling approaches have been developed. Recently, non-statistical methods and techniques [2][3] have been applied to detect and predict the changes in the region of non-linear time series, like grey system, artificial neural network (ANN) and fuzzy logic systems that can find the characteristic of complex and random data and build accurate time series models, especially of grey system that is more effective for a data set with poor message [4].

Deng [5] first proposed the grey system to build a forecasting model according to real time series of controlling system. Grey system is used to study the object that 
only presents a small part of information in the whole, and then deduce and obtain unknown or new information to develop the system. All that can be done is to find out some regular patterns from the data of time series which is called grey forecasting. The grey forecasting based on GM $(1,1)$ model [6] can try describing those uncertain parameters which are important but lack measurable messages by grey parameters and grey series. The solution to grey differential equation of the GM $(1,1)$ is an exponential function which is appreciate for the fitting of more stationary data but not fit for the fitting of data with seriously random fluctuation. The precision for prediction will be decreasing when it is used to handle the data with great fluctuation which results in many limitations in some fields. Thus, many researchers proposed new methods to improve the GM $(1,1)$ model. Tien [7] did the research on the prediction of machining accuracy by the deterministic grey dynamic model DGDM $(1,1,1)$. He [8] used greymarkov forecasting model for the electric power requirement in China. Liu [9] improved the stability of grey discrete-time systems.

Wavelet transform has been studied for many years by mathematicians and widely used in numerous applications. The wavelet transform is performed using translated and dilated versions of a single function, which is called a wavelet. It is a mathematical process that cut up data into different frequency components, and then study each component with a resolution matched to its scale. Because the signal becomes simpler in different frequency components, and is also smoothed by wavelet decomposition, the stationary of signals is better than that in non-decomposition. For the data with seriously random fluctuation, it is considered to use GM $(1,1)$ model after it is processed by wavelet decomposition. Then the conventional grey forecasting can be used to predict these data series.

In this study, a new time series forecasting model technique the GM $(1,1)$ with wavelet transform was proposed, and the application of this forecasting model was also presented. In addition, the other two models including traditional GM $(1,1)$ and ARIMA were evaluated on the basis of their efficiency to provide accurate fits and operational forecasts on the history data of energy source consumption and production in China.

\section{Research Methodologies}

\subsection{The ARIMA Model}

Box and Jenkins [10] proposed an $\operatorname{ARIMA}(p, d, q)$ model which considers the last $p$ known values of the series as well as $q$ of the past modeling errors as follows:

$$
y_{t}=\sum_{i=1}^{p} \phi_{i} y_{t-i}+\sum_{j=1}^{q} \theta_{j} e_{t-j}+\varepsilon_{t}
$$

First or second order differencing by $d$-times processes the problem of nonstationary mean, and logarithmic or power transformation of original data processes non-stationary variance.

To evaluate the appropriateness of the selected ARIMA model, the 'portmanteau' test $Q$, was also considered by calculating the statistical quantity $Q$ as follows: 


$$
\begin{gathered}
\hat{\rho}_{k}\left(e_{t}\right)=\frac{\sum_{t=1}^{N-k} e_{t} e_{t+k}}{\sum_{t=1}^{N} e_{t}^{2}} \\
Q=[N-D-\max (p, q)] \sum_{k=1}^{m} \hat{\rho}_{k}^{2}\left(e_{t}\right)
\end{gathered}
$$

In the ARIMA $(p, d, q)$ model, $Q$ follows a $\chi^{2} \alpha(m-p-q)$ distribution. When $Q \leq \chi^{2} \alpha(m-p-q)$, the ARIMA $(p, d, q)$ model is appreciate and acceptable, otherwise the model is not valid and needs reformulation.

\subsection{Wavelet Transform}

Theoretical Background. The decomposition of signals is an important procession in Wavelet transforms, especially for the analysis of non-stationary time series. There are many algorithms of wavelet decomposition, such as Mallat [11] algorithm, which has been proposed to compute the discrete wavelet transform (DWT) coefficients. It needs two extractions from signals and leads to the reduction of signal spots. It is very disadvantage to be used in prediction. However, Wickerhauser [12] considered if the input discrete $f[n]$ has $2^{L}(L \in N)$ nonzero samples. There are $L$ different scales, and each scale sequence and wavelet sequence's length are decimated by two with change of scale. Thus, this paper adopt a simple, quick algorithm called à trous algorithm without signal extraction [13]. By à trous algorithm, the sequence's length of decomposed time series won't change. What's more, the length of decomposed series in scales is equal to the length of original series. This algorithm overcomes the problem from Mallat algorithm. Besides, it is good for the reconstruction of decomposed series.

The à Trous Wavelet Transform. It is supposed that there is a time series $x(t)(t=1,2, \ldots, N)$ to be processed by wavelet decomposition, where $N$ is the present time-point. It is arranged as $c_{0}(t)=x(t)$. The decomposition with à trous algorithm is as followed:

$$
\begin{gathered}
c_{i}(t)=\sum_{k=-\infty}^{+\infty} h(k) c_{i-1}\left(t+2^{i} k\right)(i=1,2, \ldots) \\
w_{i}(t)=c_{i-1}(t)-c_{i}(t)(i=1,2, \ldots)
\end{gathered}
$$

In the formula, $h(k)$ is a discrete low-pass filter. $c_{i}(t)$ and $w_{i}(t)(i=1,2, \ldots, J)$ are the scaling coefficients and wavelet coefficients of scale $i$, and $J$ is scale number. The number of different scales is under $\log (N)$ ( $N$ is the length of time series). $\left\{w_{1}, w_{2}, \ldots, w_{J}, c_{J}\right\}$ is called wavelet decomposition or wavelet transform series under scale $J$. 
Then, the decomposed wavelet was reconstructed with à trous algorithm as follows:

$$
c_{0}(t)=c_{J}(t)+\sum_{i=1}^{J} w_{i}(t)
$$

The wavelet pass filter called Haar $h=\left(\frac{1}{2}, \frac{1}{2}\right)$ was selected in à trous algorithm. Here is the derived formula for the decomposed series in corresponding space.

$$
\begin{gathered}
c_{i+1}(t)=\frac{1}{2}\left(c_{i}\left(t-2^{i}\right)+c_{J}(t)\right) \\
w_{i+1}(t)=c_{i}(t)-c_{i+1}(t)
\end{gathered}
$$

From formula (7) and (8), it is easy to find that the wavelet coefficient for any time spot can be calculated without the information after the time of $t$.

Besides, wavelet transform with à trous algorithm needs decomposed values outside signal boundaries. Although other strategies could be envisaged, we use a mirror approach $x(N-K)=x(N+K)$. This is tantamount to redefine the discrete filter associated with the scale function in the signal boundary region and to redefine the associated wavelet function in this region. It is hypothesized that future data is based on values in the immediate past. Not surprisingly there is discrepancy in fit in the succession of scales, which grows with scale as larger numbers of immediately past values are taken into account. The first values of our time series, which also constitute a boundary, can cause difficulty, but this is of no practical consequence.

\subsection{GM (1,1) Grey Forecasting Model}

It is supposed that $x^{(0)}$ is an original time series

$$
x^{(0)}=\left\{x^{(0)}(1), x^{(0)}(2), \ldots, x^{(0)}(N)\right\}
$$

and a new series is given by the accumulated generating operation (AGO)

$$
x^{(1)}=\left\{x^{(1)}(1), x^{(1)}(2), \ldots, x^{(1)}(N)\right\}
$$

where $x^{(1)}(t)=\sum_{i=1}^{t} x^{(0)}(i), t=1,2, \ldots, N$.

According equation (10), the grey generated model, based on the series $x^{(1)}=\left\{x^{(1)}(1), x^{(1)}(2), \ldots, x^{(1)}(N)\right\}$ is given by the first-order differential equation

$$
\frac{d x^{(1)}}{d t}+a x^{(1)}=u
$$


where the coefficient $a$ and grey control parameter $u$ are the model parameters to be estimated. Then the least squares solution of $x^{(0)}$ is as followed:

$$
\hat{x}^{(1)}(t+1)=\left(x^{(0)}(1)-\frac{u}{a}\right) e^{-a t}+\frac{u}{a}(t=1,2,3, \ldots)
$$

where $\hat{b}=\left[\begin{array}{l}a \\ u\end{array}\right]=\left(B^{T} B\right)^{-1} B^{T} Y, B=\left[\begin{array}{cc}-1 / 2\left(x^{(1)}(1)+x^{(1)}(2)\right) & 1 \\ -1 / 2\left(x^{(1)}(2)+x^{(1)}(3)\right) & 1 \\ \ldots & \ldots \\ -1 / 2\left(x^{(1)}(N-1)+x^{(1)}(N)\right) & 1\end{array}\right]$, $Y=\left(x^{(0)}(2), x^{(0)}(3), \ldots, x^{(0)}(N)\right)$.

According to the equation (12), $x^{(1)}$ can be predicted. By inverse accumulated generated operation (IAGO) of $\hat{x}^{(1)}$, the forecasting value can be reversed.

$$
\hat{x}^{(0)}(t+1)=\hat{x}^{(1)}(t+1)-\hat{x}^{(1)}(t)(t=1,2,3, \ldots)
$$

where $\hat{x}^{(0)}(t)(t=1,2, \ldots, N)$ is the regression value of original data series $x^{(0)}(t)(t=1,2, \ldots, N)$, and $\hat{x}^{(0)}(t)(t>N)$ is the predicting value of original data series.

\subsection{GM (1,1) Grey Forecasting Model with Wavelet Transform}

There is a time series $x(t)(1,2, \ldots, N)$. It is processed by wavelet decomposition with à trous algorithm. And then the time series in every layer is reconstructed. It can be showed

$$
x=w_{1}+w_{2}+\ldots+w_{J}+c_{J}
$$

In this equation, $w_{i}:\left\{w_{i}(1), w_{i}(2), \ldots, w_{i}(N)\right\}$ is detail signals in the decomposed layer i. $c_{J}:\left\{c_{J}(1), c_{J}(2), \ldots, c_{J}(N)\right\}$ is the approaching signals in the decomposed layer $J$. Thus, $x(t)=w_{1}(t)+w_{2}(t)+\ldots+w_{J}(t)+c_{J}(t)$, where $x(t)$ is known at the time of $\{t \mid t<N\}$. The value next time can be obtained (equation (15)).

$$
x(t+1)=w_{1}(t+1)+w_{2}(t+1)+\ldots+w_{J}(t+1)+c_{J}(t+1)
$$

The steps of prediction for $w_{1}(t+1), w_{2}(t+1), \ldots, w_{J}(t+1), c_{J}(t+1)$ are as followed.

First, move the difference of information parallel in time series for every layer of $w_{i}$ and $c_{J}$. Then, the series moved is used to evaluate the parameters in $\operatorname{GM}(1,1)$. After moving, $w_{1}(t+1), w_{2}(t+1), \ldots, w_{J}(t+1), c_{J}(t+1)$ is predicted by GM $(1,1)$ 
model. The value of forecasting is $\hat{w}_{1}(t+1), \hat{w}_{2}(t+1), \ldots, \hat{w}_{J}(t+1), \hat{c}_{J}(t+1)$. Finally, the predicting value of original time series $x$ is obtained,

$$
\hat{x}(t+1)=\hat{w}_{1}(t+1)+\hat{w}_{2}(t+1)+\ldots+\hat{w}_{J}(t+1)+\hat{c}_{J}(t+1)
$$

\section{Examples}

A new forecasting model GM $(1,1)$ with wavelet transform, traditional GM $(1,1)$ model and ARIMA model proposed in this paper were applied to forecast energy source consumption and production in China. The duration considered in this study ranges from 1985 to 2002 . These data shown in Table 1 were refereed from National Bureau of Statistic of China [14].

Energy source consumption and production are influenced by many factors, including the economy development, industry structure, weather, policy and so on. It was shown that the time series of energy source consumption and production in a country have serious random fluctuation. However, in order to harmonize the relationship between the high needs and reduction of energy source, it is more and more important to a country to make a prediction for energy source consumption and production in the future. From Table 1, it was found that the data were rising year by year, while fluctuating randomly.

Table 1. Energy source consumption and production of China from 1985 to 2002 (unit: ten million kilogram standard coal)

\begin{tabular}{cccccc}
\hline Year & $\begin{array}{c}\text { Energy source } \\
\text { consumption }\end{array}$ & $\begin{array}{c}\text { Energy source } \\
\text { production }\end{array}$ & Year & $\begin{array}{c}\text { Energy source } \\
\text { consumption }\end{array}$ & $\begin{array}{c}\text { Energy source } \\
\text { production }\end{array}$ \\
\hline 1985 & 76682 & 85546 & 1994 & 122737 & 118729 \\
1986 & 80850 & 88124 & 1995 & 131176 & 129034 \\
1987 & 86632 & 91266 & 1996 & 138948 & 132616 \\
1988 & 92997 & 95801 & 1997 & 137798 & 132410 \\
1989 & 96934 & 101639 & 1998 & 132214 & 124250 \\
1990 & 98703 & 103922 & 1999 & 130119 & 109126 \\
1991 & 103783 & 104844 & 2000 & 130297 & 106988 \\
1992 & 109170 & 107256 & 2001 & 134914 & 120900 \\
1993 & 115993 & 111059 & 2002 & 148222 & 138369 \\
\hline
\end{tabular}

To eliminate the fluctuation of these two data sets, wavelet transform with à trous algorithm was used to data processing before GM $(1,1)$. Fig. 1 shows the decomposed signals of low and high frequency in two layers. The non-stationary time series data was smoothed and parallel shifted by wavelet decomposition, and the fluctuation was considered noises decomposed into high frequency. The detail signals and approaching signals were used for wavelet reconstruction. 


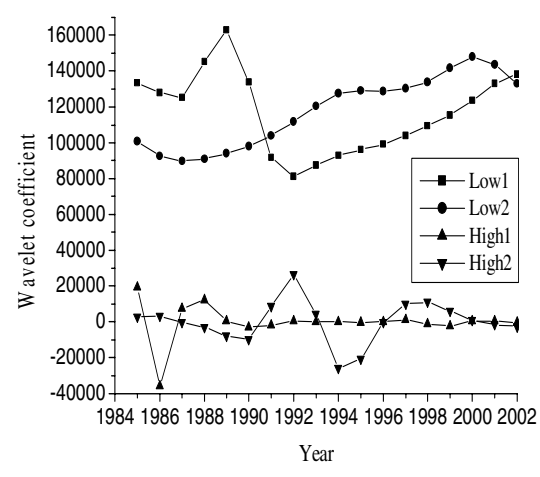

(a)

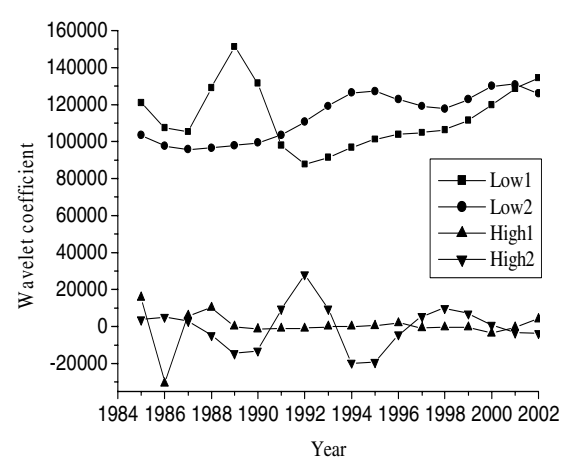

(b)

Fig. 1. Detail signals of (a) energy source consumption and (b) energy source production in low and high frequency of two layers with wavelet decomposition

In this study, wavelet transform was performed in software Matlab 7.0, and GM $(1,1)$ and ARIMA model were achieved in DPS, a software of data processing system for Practical Statistics [1]

The forecasting results for energy source consumption and production from 2000 to 2002 by GM $(1,1)$ with wavelet transform were shown in Table 2. Meanwhile, the forecasting values from traditional GM $(1,1)$ and ARIMA were also obtained. In these three models, GM $(1,1)$ with wavelet transform displayed the highest precision except one year forecasting on energy source production. The data without any processing used in GM $(1,1)$ gave the lowest precision, which revealed the default of GM $(1,1)$ model for non-stationary series only using poor information to forecast. Although ARIMA model seems better than GM $(1,1)$, it needs more history data. For practical application, most of the forecasting problems are described under poor information in a data set, or even a small data. Thus, the improved GM $(1,1)$ based on wavelet transform is promising for non-stationary or lack information time series forecasting.

Table 2. Forecasting results from three different models for the energy source consumption and production from 2000 to 2002 (unit: ten million kilogram standard coal)

\begin{tabular}{cccccc}
\hline \multicolumn{2}{c}{ Wavelet-GM $(1,1)$} & \multicolumn{2}{c}{ GM $(1,1)$} & \multicolumn{2}{c}{ ARIMA } \\
\hline \multirow{3}{*}{ Prediction } & Precision & Prediction & Precision & Prediction & Precision \\
136141 & $95.71 \%$ & 149483 & $87.17 \%$ & 140261 & $92.90 \%$ \\
139278 & $96.87 \%$ & 155455 & $86.79 \%$ & 128887 & $95.53 \%$ \\
143761 & $96.99 \%$ & 161665 & $91.68 \%$ & 135567 & $91.46 \%$ \\
114161 & $93.72 \%$ & 133790 & $79.97 \%$ & 103809 & $97.03 \%$ \\
120226 & $99.44 \%$ & 137300 & $88.06 \%$ & 112351 & $92.93 \%$ \\
126715 & $98.20 \%$ & 126716 & $91.58 \%$ & 113291 & $81.88 \%$ \\
\hline
\end{tabular}




\section{Conclusions}

Grey forecasting model is a good method for the time series changed smoothly. However, the predicting precision will reduce when it is used to forecast time series with serious random fluctuation. The non-stationary time series are influenced by many factors, which make the prediction more complexly. The wavelet transform is an effective tool for time history dynamic analysis of structures. The problem in GM $(1,1)$ model can be solved by wavelet analysis, which can decompose the time series into different layers according to the different scales. As the case study shows that the accuracy of GM $(1,1)$ model with wavelet transform in forecasting energy source consumption and production from 2000 to 2002 is higher than these in traditional GM $(1,1)$ and ARIMA models. Thus, it is concluded that GM $(1,1)$ model with wavelet transform is promising for time series analysis, especially for non-stationary time series.

Besides, there is a problem in wavelet decomposition. The selection of algorithm in wavelet decomposition is very important for the result of wavelet analysis. In this paper, a simple and quick algorithm called à trous algorithm without signal extraction was used in wavelet decomposition. By à trous algorithm, the length of decomposed series in scales is equal to the length of original series. This algorithm overcame the problem from Mallat algorithm. However, wavelet transform with à trous algorithm need decomposed values outside signal boundaries. Although other strategies could be envisaged, we used a mirror approach. It is necessary to study further whether this method is popular for any time series.

\section{Acknowledgments}

This study was supported by the Teaching and Research Award Program for Outstanding Young Teachers in Higher Education Institutions of MOE, P. R. C., Natural Science Foundation of China (Project No: 30270773), Specialized Research Fund for the Doctoral Program of Higher Education (Project No: 20040335034), and Science and Technology Department of Zhejiang Province (Project No. 2005C21094, 2005C12029).

\section{References}

1. Tang, Y.M., Feng, M.G.: Data Processing System for Practical Statistics. Science Publishing Company Press. Beijing (2002)

2. Wu, B., Chen, M.H.: Use of Fuzzy Statistical Technique in Change Periods Detection of Nonlinear Time Series. Applied Mathematics and Computation. Vol. 99. No. 2-3 (1999) 241-254

3. He, Y., Zhang, Y., Xiang, L.G.: Study of Application Model on BP Neural Network Optimized by Fuzzy Clustering. Lecture Notes in Artificial Intelligence. Vol. 3789 (2005) 712-720

4. Deng, J.L.: Control Problems of Grey System. Huazhong University of Science and Technology Press. Wuhan (1990) 1-2

5. Deng, J.L.: Grey Forecasting and Decision Making. Huazhong University of Science and Technology Press. Wuhan (1985) 
6. Bao, Y.D., Wu, Y.P., He, Y.: A New Forecasting Model Based on the Combination of GM $(1,1)$ Model and Linear Regression. Systems Engineering (Theory and Practice). Vol. 24. No. 3 (2004) 95-98

7. Tien, T.L.: A Research on the Prediction of Machining Accuracy by the Deterministic Grey Dynamic Model DGDM (1,1,1). Applied Matheatics and Computation. Vol. 161. No. 3 (2005) 923-945

8. He, Y., Huang, M.: A Grey_Markov Forecasting Model for the Electric Power Requirement in China. In:Gelbukh, A., Albornoz, A.D., Terashima, H.(eds.): Lecture Notes in Artificial Intelligence. Vol. 3789 (2005) 574-582

9. Liu, P.L., Shyr, W.J.: Another Sufficient Condition for the Stability of Grey Discrete-time Systems. Journal of the Franklin Institute. Vol. 342. No. 1 (2005) 15-23

10. Box, G.E.P., Jenkins, G.M.: Time Series Analysis. Forecasting and Control. Holden-Day. San Francisco (1976) 575

11. Li, X.B., Li, H.Q., Wang, F.Q., Ding, J.: A Remark on the Mallat Pyramidal Algorithm of Wavelet Analysis. Communications in Nonlinear Science and Numerical Simulation. Vol. 2. No. 4 (1997) 240-243

12. Wickerhauser, M.V.: Adapted Wavelet Analysis from Theory to Software. Wellesley. Massachusetts (1994) 213-235

13. Sun, Z.M., Jiang, X.W., Wang, X.F.: Application of à Trous Wavelet to Satellite Telemetry Data Recursive Prediction. Journal of Nanjing University of Science and Technology. Vol. 28. No. 6 (2004) 606-611

14. National Bureau of Statistic of China: China Statistical Year Book. China Statistics Press. Beijing (2004) 